\title{
PUMP-PUMP FOUR-WAVE MIXING IN DISTRIBUTED FIBER RAMAN AMPLIFIERS
}

\section{MEZCLA DE CUATRO ONDAS ENTRE BOMBAS EN AMPLIFICADORES DE FIBRA RAMAN DISTRIBUIDOS}

\author{
Marcelo Soto $^{1} \quad$ Ricardo Olivares ${ }^{1}$ \\ Recibido 28 de agosto de 2006, aceptado 18 de junio de 2007 \\ Received: August 28, $2006 \quad$ Accepted: June 18, 2007
}

\begin{abstract}
RESUMEN
En este trabajo se presenta un modelo matemático que describe rigurosamente la interacción entre esparcimiento Raman estimulado (SRS) y mezcla de cuatro ondas (FWM) en amplificadores Raman distribuidos (DFRAs). Los efectos paramétricos ocurridos en DFRAs debido al proceso de FWM entre bombas, son completamente caracterizados. Los resultados de las simulaciones numéricas son contrastados con mediciones experimentales llevadas a cabo en diferentes configuraciones de DFRAs. Los resultados permiten validar el modelo matemático propuesto, el cual puede ser extendido para incluir otras no-linealidades. Finalmente, se analizan los efectos de FWM entre bombas en el comportamiento espectral de la ganancia de un DFRA.
\end{abstract}

Palabras clave: Amplificador de fibra Raman distribuido (DFRA), mezcla de cuatro ondas (FWM), redes ópticas WDM, ecualización de potencia.

\section{ABSTRACT}

In this work, a comprehensive mathematical model which rigorously describes the interaction between stimulated Raman scattering (SRS) and four-wave mixing (FWM) in distributed fiber Raman amplifiers (DFRAs) is presented. The parametric effects on DFRAs due to pump-pump FWM processes are completely characterized. Numerical simulations are contrasted with experimental measurements which were carried out with different configurations of DFRAs. The results validate the proposed mathematical model, which can be extended to include other non-linear effects. Finally, the pump-to-pump FWM effects on the spectral gain of a DFRA are analyzed.

Keywords: Distributed Fiber Raman Amplifier (DFRA), Four-Wave Mixing (FWM), WDM optical networks, power equalization.

\section{INTRODUCTION}

Distributed Fiber Raman Amplifiers (DFRAs) have emerged as promising devices in extending the span length and transmission capacity of wavelength division multiplexed (WDM) optical networks. The advantages of DFRAs compared to Erbium-Doped Fiber Amplifiers (EDFAs) are related to design flexibility, broad gain bandwidth, low noise and its capacity to reduce the effect of fiber nonlinearities [1]. However, the good performance of a DFRA is dramatically determined by the mathematical model and the method used to design it $[2,3]$. DFRAs working with low chromatic dispersion fibers or with pumping waves located near the zero dispersion wavelength $\left(\lambda_{Z D}\right)$ require the development of numerical models that include other nonlinearities such as four wave mixing (FWM) [4-6].

In this publication a mathematical model including the interaction of SRS and FWM in a single differential equation is presented. The effects of this interaction have been studied separately, but recent work carried out by other researchers have taken both aspects of the model into account, either by using analytical approximations or iterative numerical methods [6-10]. The model developed in this work numerically solves the interaction of both nonlinearities for co-propagating signals in a straightforward way. It is validated by comparing simulation results with experimental measurements using DFRAs with different features. Finally, the impact of FWM on

\footnotetext{
1 Universidad Técnica Federico Santa María. Departamento de Electrónica. Casilla Postal 110-V. Av. España 1680. Valparaíso, Chile.
} Email:msotoh@elo.utfsm.cl,rov@elo.utfsm.cl 
a gain-equalized DFRA is rigorously analyzed by using the proposed model.

\section{THEORETICAL MODEL DESCRIPTION}

The model to be developed should consider the Raman interactions present in the fiber such as gain and depletion due to SRS. It should also include effects of parametric gain, depletion, generation of new waves and the coherent superposition of electric fields at identical frequency occurring, due to FWM products of the pumps and WDM channels [11]. Considering the above mentioned effects, the behavior of the amplitude and phase of all the propagated waves must be taken into account in the proposed mathematical model.

If signals having different wavelengths are introduced into the fiber, a general expression for the evolution of the complex envelope of the normalized electric field $A_{F}(z)$, at frequency $f_{F}$, can be obtained from the inhomogeneous propagation equation presented in [11]:

$$
\begin{aligned}
\frac{d A_{F}(z)}{d z}= & -\frac{\alpha_{F}}{2} A_{F}(z) \\
+j \frac{1}{3} & \gamma \sum_{i, j \neq k}\left\{K_{p o l}\left(f_{i}, f_{j}, f_{k}\right) D_{i j k}\right. \\
& \left.\times A_{i}(z) A_{j}(z) A_{k}^{*}(z) \exp \left(-\Delta \beta_{i j k} z\right)\right\} \\
+j \frac{1}{3} \gamma & \sum_{p, r \neq q}\left\{K_{p o l}\left(f_{F}, f_{q}, f_{r}\right) D_{p q r}\right. \\
& \left.\times A_{p}(z) A_{q}^{*}(z) A_{r}(z) \exp \left(\Delta \beta_{p q r} z\right)\right\}
\end{aligned}
$$

where $\alpha_{F}$ is the attenuation coefficient of the fiber at frequency $f_{F} ; \gamma$ is the nonlinear coefficient; $D_{i j k}$ is the degeneracy factor; $\Delta \beta_{i j k}=\beta_{i}+\beta_{j}-\beta_{k}-\beta_{F}$ is the linear phase-mismatch factor; and $K_{\text {pol }}\left(f_{i}, f_{j}, f_{k}\right)$ is the polarization factor for FWM defined in [12]. It is 1 when the waves involved in the FWM process have parallel linear polarizations, and $(1 / 2)^{1 / 2}$ or $(3 / 8)^{1 / 2}$ for partially degenerate and non-degenerate FWM with random polarization states, respectively.

The first right hand side term of equation (1) represents the attenuation of $A_{F}(z)$ due to fiber losses. The second term describes the parametric behavior of this wave due to generation of FWM products and parametric gain generated from all the electric field combinations of frequencies $f_{i}, f_{j}$ and $f_{k}$ (with $i, j \neq k$ ) that satisfied the relation $f_{F}=f_{i}+f_{j}-f_{k}$. The third term describes the depletion experimented by $A_{F}(z)$ due to the FWM process for all frequency combinations $f_{p}, f_{q}$ and $f_{r}$ (with $F, q \neq r$ ) where the condition $f_{p}=f_{F}+f_{q}-f_{r}$ is satisfied. This equation generalizes the well-known FWM model derived in [11], and it can be applied to any wave acting in the FWM process, e.g. FWM pump waves, signal waves and FWM products (also called idler waves).

If the gain and depletion, due to SRS are included in a similar form as the expression representing the fiber loss in the above mentioned inhomogeneous equation, and the polarization states of the interacting waves are considered, a single differential equation that completely describes the SRS and FWM effects in a coupled form can be derived, as in equation (2):

$$
\begin{aligned}
\frac{d A_{F}(z)}{d z}= & -\frac{\alpha_{F}}{2} A_{F}(z) \\
+ & \sum_{f_{m}>f_{F}} \frac{g_{R}\left(f_{F}, f_{m}\right)}{2 A_{e f f} K_{e f f}\left(f_{F}, f_{m}\right)}\left|A_{m}(z)\right|^{2} A_{F}(z) \\
- & \sum_{f_{m}<f_{F}} \frac{f_{F}}{f_{m}} \frac{g_{R}\left(f_{F}, f_{m}\right)}{2 A_{e f f} K_{e f f}\left(f_{F}, f_{m}\right)}\left|A_{m}(z)\right|^{2} A_{F}(z) \\
+ & j \frac{1}{3} \gamma \sum_{i, j \neq k}\left\{K_{p o l}\left(f_{i}, f_{j}, f_{k}\right) D_{i j k}\right. \\
& \left.\times A_{i}(z) A_{j}(z) A_{k}^{*}(z) \exp \left(-\Delta \beta_{i j k} z\right)\right\} \\
+ & j \frac{1}{3} \gamma \sum_{p, r \neq q}\left\{K_{p o l}\left(f_{F}, f_{q}, f_{r}\right) D_{p q r}\right. \\
& \left.\times A_{p}(z) A_{q}^{*}(z) A_{r}(z) \exp \left(\Delta \beta_{p q r} z\right)\right\}
\end{aligned}
$$

where $K_{\text {eff }}\left(f_{F} f_{m}\right)$ is the polarization factor of the waves at frequency $f_{F}$ and $f_{m}$ for Raman interaction, which is either 0.5 (identical polarization) or 1 (random polarization) [4]. Equation (2) shows the evolution of the electric field associated with all signals present in the SRS-FWM interaction, e.g. the Raman pump sources, WDM channels and FWM products.

One of the main advantages of this approach is that the model can be straightforwardly solved by using a simple numerical technique, thus avoiding the solution of SRS and FWM separately. Note, that when both models are solved independently, an iterative procedure is required in order to get convergence in solution [6]. Therefore, each model should be solved several times, depending on the degree of interaction between SRS and FWM processes, which is given by the involved wavelengths, powers and $\lambda_{Z D}$. Thus, the proposed model allows one to reduce the computing time due to the straightforward way of solving it. This effect can be mainly noted when strong interactions among both nonlinearities take place because a long computing time is required to solve both models iteratively. In addition, this model avoids the use of closed mathematical expressions based on undepleted 
conditions, which are inappropriate approximations when high SRS-FWM interactions exist and/or when many optic waves are propagated along the fiber. To our best knowledge, equation (2) corresponds to a new model and it is the major contribution of this publication.

\section{NUMERICAL SOLUTION OF THE MODEL}

The proposed model describes the propagation of electric field waves in terms of a system of complex differential equations. If $N$ signals with different wavelengths (pumps and channels) are introduced into the fiber, $\left(N^{3}-N^{2}\right) / 2$ FWM products will be generated. Therefore, in the worst case, a system of $\left(N^{3}-N^{2}\right) / 2+N$ differential equations will be obtained, each one similar to (2) for each propagated wave frequency. This system of equations can be solved by a simple numerical technique. In this work, we use the fourth order Runge-Kutta method to solve it, separating equation (2) in two parts: one, related with the magnitude of the electric field, and the other associated with the phase of the electric field. Thus, from (2) the following expressions are obtained:

$$
\begin{aligned}
& \frac{d\left|A_{F}(z)\right|}{d z}=-\frac{\alpha_{F}}{2}\left|A_{F}(z)\right| \\
& +\sum_{f_{m}>f_{F}} \frac{g_{R}\left(f_{F}, f_{m}\right)}{2 A_{e f f} K_{e f f}\left(f_{F}, f_{m}\right)}\left|A_{m}(z)\right|^{2}\left|A_{F}(z)\right| \\
& -\sum_{f_{m}<f_{F}} \frac{f_{F}}{f_{m}} \frac{g_{R}\left(f_{F}, f_{m}\right)}{2 A_{e f f} K_{e f f}\left(f_{F}, f_{m}\right)}\left|A_{m}(z)\right|^{2}\left|A_{F}(z)\right| \\
& -\frac{1}{3} \gamma \sum_{i, j \neq k}\left\{K_{p o l}\left(f_{i}, f_{j}, f_{k}\right) D_{i j k}\right. \\
& \left.\quad \times\left|A_{i}(z)\right|\left|A_{j}(z)\right|\left|A_{k}(z)\right| \sin \left(\Delta \beta_{i j k} z+\phi_{i j k}(z)\right)\right\} \\
& -\frac{1}{3} \gamma \sum_{p, r \neq q}\left\{K_{p o l}\left(f_{F}, f_{q}, f_{r}\right) D_{p q r}\right. \\
& \left.\quad \times\left|A_{p}(z)\right|\left|A_{q}(z)\right| A_{r}(z) \mid \sin \left(-\Delta \beta_{p q r} z+\phi_{p q r}(z)\right)\right\} \\
& \frac{d \phi_{F}(z)}{d z}=\frac{1}{3} \gamma \sum_{i, j \neq k}\left\{K_{p o l}\left(f_{i}, f_{j}, f_{k}\right) D_{i j k}\right. \\
& \quad \times \frac{\left|A_{i}(z)\right|\left|A_{j}(z)\right|\left|A_{k}(z)\right|}{3} \sum_{p, r \neq q}\left\{K_{p o l}\left(f_{F}, f_{q}, f_{r}\right) D_{p q r}(z)|| A_{q}(z)\left|A_{r}(z)\right|\right. \\
&
\end{aligned}
$$

Where:

$$
\begin{aligned}
& \phi_{i j k}(z)=\phi_{i}(z)+\phi_{j}(z)-\phi_{k}(z)-\phi_{F}(z) \\
& \phi_{p q r}(z)=\phi_{p}(z)+\phi_{r}(z)-\phi_{q}(z)-\phi_{F}(z)
\end{aligned}
$$

The effect of FWM on the amplitude and phase of the propagated waves can be observed in equations (3) and (4), respectively. However, the absorption losses and SRS only affect the amplitude of the electric field.

\section{COMPARATIVE ANALYSIS OF THE MODEL}

We compare results obtained from equation (2) with those obtained with the SRS model developed in [2] and [4]. Then, we compare results obtained applying equation (2) with the FWM model presented in [13]. Special care has to be taken since the SRS and the FWM model are developed in terms of power equations, while equation (2) is expressed in terms of electric field. For each case, the nonlinear effect that is not considered by the SRS or FWM model has to be omitted in equation (2).

\section{Modeling SRS effects only}

The objective of this subsection is to compare numerical results obtained by using equation (2) with results obtained when the classical SRS models [2,4] are solved. The simulations take into account the propagation of 100 WDM channels with wavelengths located in the range [1520-1600] nm, separated by $0.8 \mathrm{~nm}$, and with $0.5 \mathrm{~mW}$ of input power. The analyzed DFRA is implemented on a standard single mode fiber (SSMF) of $50 \mathrm{~km}$ length, with an $A_{\text {eff }}=95 \mu \mathrm{m}^{2}$, and it is pumped by using one source at $1450 \mathrm{~nm}$ with $350 \mathrm{~mW}$. Fig. 1 shows the output power obtained for 3 different cases considering only SRS interactions in equation (2) and neglecting effects of FWM. Case 1 shows the output power obtained by using the model presented in [2], which only considers SRS interactions. Case 2 corresponds to the results obtained by the model presented in [4], which in addition to SRS, also includes gain and depletion due to spontaneous Raman scattering (Stokes and anti-Stokes scattering). In this case, the frequency spectrum has been partitioned by $0.2 \mathrm{~nm}$ to simulate the propagation of the amplified spontaneous emission (ASE) noise. However, since the noise effect is negligible (output signal-to-noise ratio higher than $30 \mathrm{~dB}$, taking into account ASE noise and Rayleigh scattering), both curves (Case 1 and Case 2) are coincided for all the considered channels. Case 3 corresponds to 
the results obtained by solving equation (2), neglecting the FWM effects. In figure 1, it is possible to verify a good agreement between the 3 cases at the fiber output. Numerical differences among all 3 cases are less than $0.28 \%$. Therefore, we can say that the evaluation of (2) produces similar results as those obtained by using the models presented in [2] and [4] under identical parameter configurations.

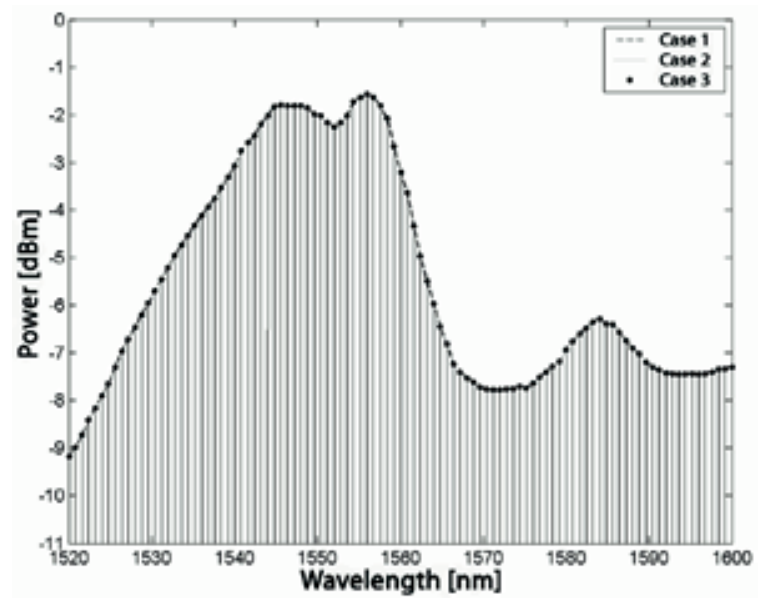

Figure 1. Simulation of DFRA with 1 pump and 100 WDM channels, solving different models: Case 1, solving model presented in [2]; Case 2, solving model presented in [4]; Case 3, solving new proposed model (neglecting FWM interactions).

\section{Modeling FWM effects only}

In this subsection we compare the results obtained by equation (2) when only FWM processes are taken into account. In order to validate the modeling of parametric gain, depletion due to FWM and the generation of FWM products, equation (2) is solved neglecting SRS terms. Numerical results are compared with the analytical expression presented in [13], which are valid only when the condition $\Delta \beta=0$ is satisfied:

$$
\begin{gathered}
P_{1}(z)=P_{P}(0) \cdot y \cdot \exp (-\alpha z) \\
P_{2}(z)=P_{P}(0) \cdot y \cdot \exp (-\alpha z) \\
P_{3}(z)=\left[P_{P}(0) \cdot(1-y)+P_{3}(0)\right] \cdot \exp (-\alpha z) \\
P_{4}(z)=\left[P_{P}(0) \cdot(1-y)\right] \cdot \exp (-\alpha z)
\end{gathered}
$$

Where $P_{P}(0)=P_{1}(0)=P_{2}(0)$ correspond to the input pump powers of the FWM process; $P_{3}(0)$ is the input power of the signal wave, which is affected by parametric gain; and $y$ is a variable defined as follows:

$$
\begin{gathered}
y=\frac{r+1}{r \cdot \cosh ^{2}(f \sqrt{r+1})+1} \\
r=\frac{P_{3}(0)}{P_{P}(0)} \\
f=2 \gamma \cdot P_{P}(0) \frac{[1-\exp (-\alpha z)]}{\alpha}
\end{gathered}
$$

To carry out the comparison, the used parameters of the fiber were $\alpha=0.23 \mathrm{~dB} / \mathrm{km}, A_{\text {eff }}=70 \mu \mathrm{m}^{2}$, and $\gamma=1.853 \times 10^{-5} \mathrm{~cm}^{-1} \mathrm{~W}^{-1}$. Note that the phase-match condition was imposed independent of the involved wavelengths (frequencies are taken into account in the calculation of $\Delta \beta$ as shown in equation (15), however the condition of $\Delta \beta=0$ was imposed for all wavelength). Based on the FWM efficiency ( $\eta$ in equation (14)), we can note that this condition allows one to compare the results when a strong FWM interaction exists:

$$
\eta=\frac{\alpha^{2}}{\alpha^{2}+\Delta \beta^{2}}\left[1+\frac{4 e^{-\alpha L} \sin ^{2}(\Delta \beta L / 2)}{\{1-\exp (-\alpha L)\}^{2}}\right]
$$

Where $\alpha$ is the attenuation coefficient; $L$ is the fiber length; and $\Delta \beta$ is the linear phase-mismatch factor, which can be obtained as follows:

$$
\begin{aligned}
\Delta \beta & =\frac{2 \pi \lambda_{0}^{2}}{c}\left\{\left(f_{i}-f_{k}\right)\left(f_{j}-f_{k}\right)\right\} D_{c}\left(f_{o}\right) \\
& -\frac{\pi \lambda_{0}^{4}}{c^{2}}\left\{\left(f_{i}-f_{k}\right)\left(f_{j}-f_{k}\right)\right\}\left\{f_{i}+f_{j}-2 f_{o}\right\} S_{c}\left(f_{o}\right)
\end{aligned}
$$

Where $f_{i}, f_{j}$ and $f_{k}$ are the frequencies involved in the FWM process, $c$ is the light velocity and, $D_{c}$ and $S_{0}$ are the chromatic dispersion and dispersion slope of the fiber at frequency $f_{0}\left(\lambda_{0}\right)$, respectively.

Figures 2(a)-(b) show power as a function of the distance for the four waves involved in the FWM process when the input powers are $12 \mathrm{dBm}$ and $15 \mathrm{dBm}$, respectively (similar conditions and results than the presented in [13]). Figures 3(a) and 3(b) show the relative error, as a function of the distance, between the numerical results obtained by solving the proposed model and the 
analytical results obtained by equations (7)-(13). We can note at the input of the fiber that the FWM product (idler wave) experiences a rather large error, but this is because it is impossible to evaluate (4) with a boundary condition of $0 \mathrm{~mW}$ at the fiber input (as it would be from a physical point of view). Setting this value to $0 \mathrm{~mW}$, a division by zero is produced. Therefore, we evaluate this expression with a sufficiently small input power (for example $\left.10^{-14} \mathrm{~W}\right)$. However, the absolute error is small enough to achieve a relative error of less than $0.1 \%$ at the fiber output. If power levels of the other waves are compared along the fiber, differences of less than $1 \%$ are obtained.

\section{EXPERIMENTAL SETUP FOR MODEL VALIDATION}

To validate the analytical model of equation (2) experimentally, four configurations of DFRAs are performed by using different types of fiber and pumping schemes as illustrated in figure 4 . The first setup, named "DFRA 1", considers only 2 pumping sources located at $1480 \mathrm{~nm}$ and $1488 \mathrm{~nm}$ with adjustable power. Six parallel WDM channels, separated each by $1.9 \mathrm{~nm}$ in the wavelength range [1581-1590.5] $\mathrm{nm}$, are linearly polarized to feed the fiber input with a power of $30 \mu \mathrm{W} /$ ch. A TrueWave fiber is used with $A_{\text {eff }}=58 \mu \mathrm{m}^{2}, \gamma=$ $2.8 \times 10^{-3} \mathrm{~m}^{-1} \mathrm{~W}^{-1}, \lambda_{Z D}=1498 \mathrm{~nm}$, and dispersion slope $S_{0}=0.05\left[\mathrm{ps} / \mathrm{km} \times \mathrm{nm}^{2}\right]$. The remaining setups, named "DFRAs 2-4", consider 3 pumping sources placed at $1450 \mathrm{~nm}, 1480 \mathrm{~nm}$ and $1488 \mathrm{~nm}$, with input power of $100 \mathrm{~mW}, 180 \mathrm{~mW}$ and $150 \mathrm{~mW}$, respectively. The 6 WDM channels are separated by $1.5 \mathrm{~nm}$ and located in the wavelength range [1570-1577.5] nm, with input power of $1 \mu \mathrm{W} / \mathrm{ch}$. DFRA 2 setup uses the same fiber as the used in DFRA 1 setup (TrueWave), but the third experiment (DFRA 3 setup) is assembled by using a Corning SMF-DS fiber, with $A_{\text {eff }}=50 \mu \mathrm{m}^{2}, \gamma=3 \times 10^{-3}$ $\mathrm{m}^{-1} \mathrm{~W}^{-1}, \lambda_{Z D}=1538 \mathrm{~nm}$ and $S_{0}=0.04\left[\mathrm{ps} / \mathrm{km} \times \mathrm{nm}^{2}\right]$. Finally, the DFRA 4 setup uses a Corning SMF-LS fiber, with $A_{\text {eff }}=55.4 \mu \mathrm{m}^{2}, \gamma=3 \times 10^{-3} \mathrm{~m}^{-1} \mathrm{~W}^{-1}, \lambda_{Z D}=1570 \mathrm{~nm}$

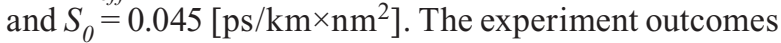
are measured with the Optical Spectrum Analyzer (OSA) illustrated in figure 4 and they are shown in figures 5 , $7-9$ as continuous black lines.

\section{RESULTS AND DISCUSSION}

Once the fibers are conveniently characterized in terms of Raman gain and losses, their parameters can be plugged into equation (2), which is solved using the fourth-order Runge-Kutta method, for the previously described conditions. Due to the low FWM efficiency of the channel-to-channel and pump-to-channel interactions, only the pump-to-pump FWM processes are considered in the solution. Also, due to the linear polarization of the channels and the depolarized used pump sources, the polarization factor considered in the simulations for channel-to-channel SRS interactions was $K_{\text {eff }}\left(f_{F} f_{m}\right)=0.5$. However, the polarization factor used for pump-to-pump and pump-to-channel interactions was $K_{\text {eff }}\left(f_{F} f_{m}\right)=1$ (in the case of SRS) and $K_{\text {pol }}\left(f_{i} f_{j} f_{k}\right)=(1 / 2)^{1 / 2}$ or $K_{p o l}\left(f_{i} f_{j} f_{k}\right)$ $=(3 / 8)^{1 / 2}$ (for partially degenerated or non-degenerated FWM).
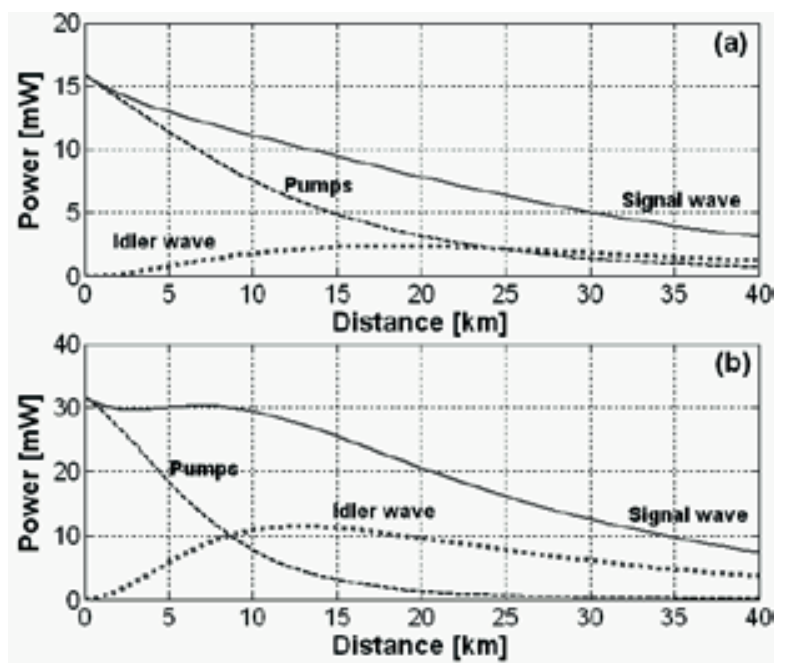

Figure 2. Numerical results obtained by using equation (2) and neglecting SRS effects. Power of the waves involved in the FWM process. (a) $\mathrm{P}_{\mathrm{IN}}$ $=12 \mathrm{dBm}$. (b) $\mathrm{P}_{\mathrm{IN}}=15 \mathrm{dBm}$.
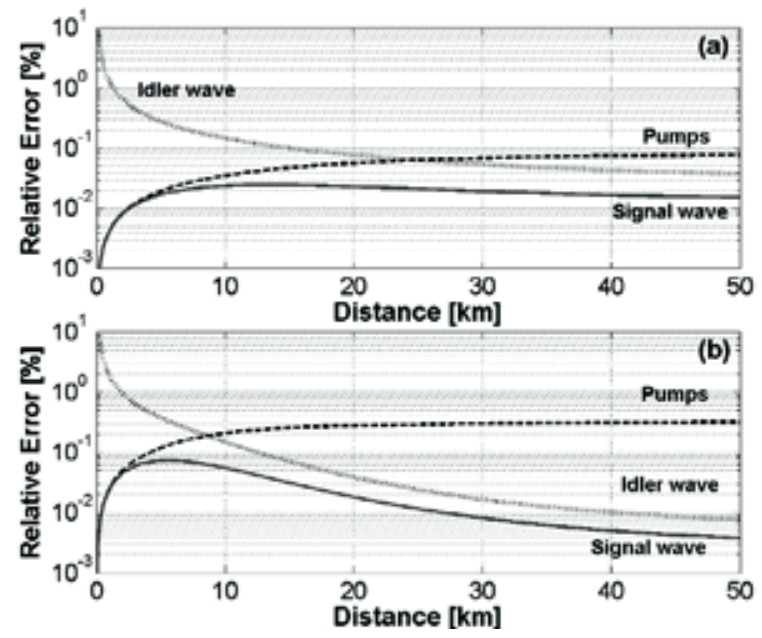

Figure 3. Relative error between numerical and analytical results. (a) $\mathrm{P}_{\mathrm{IN}}=12 \mathrm{dBm}$. (b) $\mathrm{P}_{\mathrm{IN}}=15 \mathrm{dBm}$. 


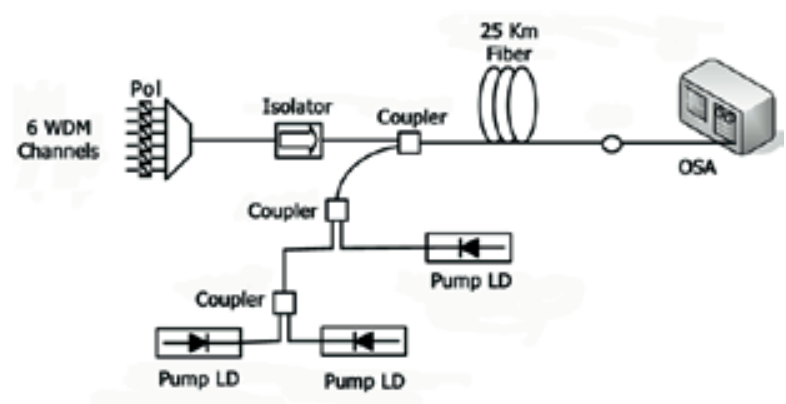

Figure 4. Experimental Setup.

Due to the phase mismatching condition present in the four experiment setups, the FWM products experiment longitudinal oscillations along the fiber [14] according to the value of $\Delta \beta$. The period and amplitude of these oscillations are related to the pump wavelengths, $\lambda_{z D}, S_{0}$, and the position $z$ along the fiber. For that reason, and because the length of the used fibers is only known to be approximately $25 \mathrm{~km}$, a direct comparison between measured and calculated power is only possible if, for each DFRA experiment, the calculated power is adjusted to be within a small power range. Thus, the outcomes of the simulated results are shown in figures 5-9 as error bars which take into account the power range obtained at $25 \mathrm{~km} \pm 100 \mathrm{~m}$ fiber distance.

In the first experiment (DFRA 1), the power of both pumps are set to be identical and varied between $100 \mathrm{~mW}$ and $250 \mathrm{~mW}$ by using $50 \mathrm{~mW}$ steps. In the output power spectrum shown in figure 5 , for $250 \mathrm{~mW}$ pump power, the two partially degenerated FWM products $\left(f_{112}\right.$ and $\left.f_{221}\right)$, generated from the pumps, can be observed. These new optical waves could act as pumps of the amplifier and modify the net Raman gain spectrum depending on the generated wavelength and the peak power achieved along the fiber. A good agreement among numerical results and measurements (wavelength and power of pumps and channels) can be observed. Differences are less than $3 \mathrm{~dB}$ and they can be attributed to the presence of other nonlinearities which are not considered in the model, such as stimulated Brillouin scattering (SBS), self-phase modulation (SPM) and cross-phase modulation (XPM). Figure 6 compares experimental and analytical outcomes of the output power of both degenerated FWM products, as a function of the input pump power. It shows differences less than $2 \mathrm{~dB}$.

For the second amplifying scheme (DFRA 2), figure 7 illustrates the high interaction between FWM and SRS due to the low chromatic dispersion around the pump wavelengths. The differences between the experimental

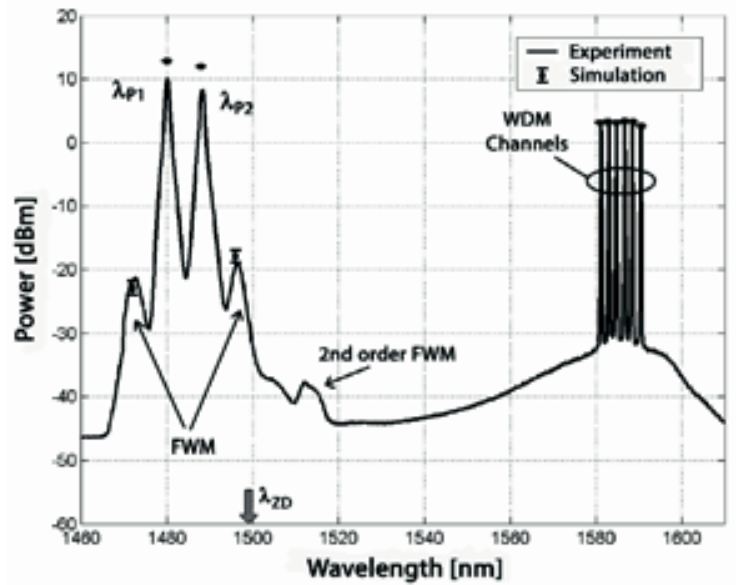

Figure 5. Experimental vs numerical results. Output power spectrum of DFRA 1 setup, with $\mathrm{P}_{\text {pump }}$ $=250 \mathrm{~mW}$.

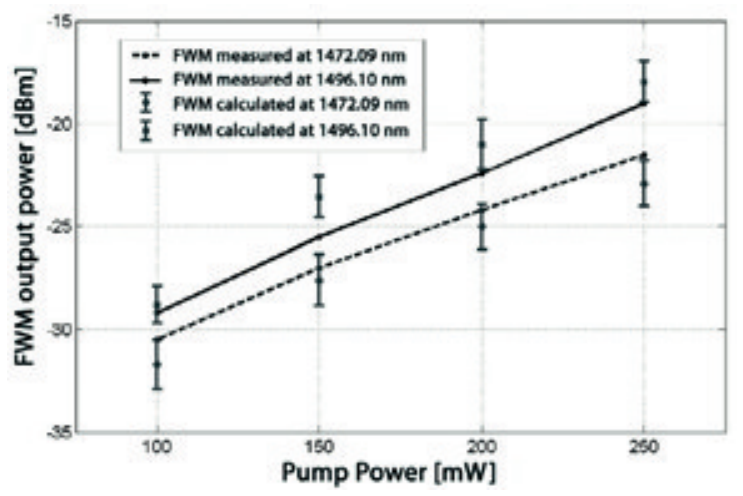

Figure 6. Measured and simulated output power of the degenerated FWM products as a function of the pump power per source (DFRA 1).

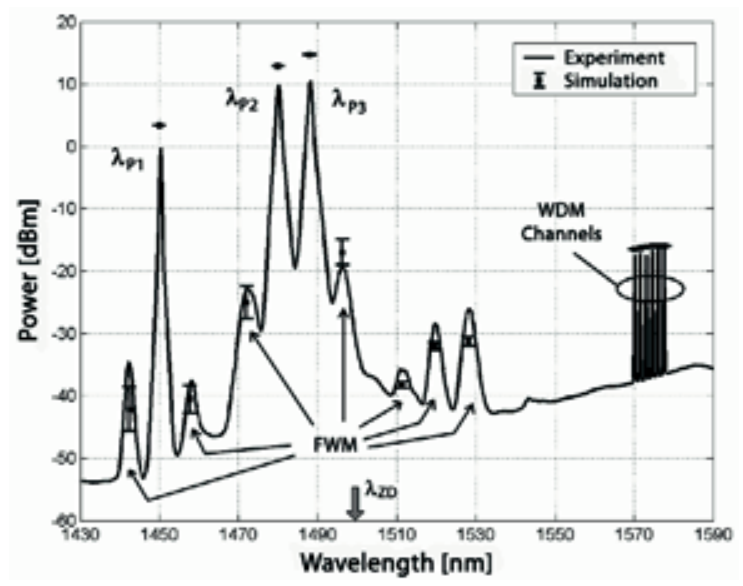

Figure 7 Simulation results vs experimental measurements. Output power spectrum of DFRA 2 setup. 
measurements and the simulation results can be attributed to longitudinal random variations of some parameters such as $A_{e f f} S_{0}$, and mainly $\lambda_{Z D}$, which have high FWM impact in low dispersion regions.

Differences among the results are also justified by the phase shift induced by SPM and XPM which change the phase mismatch condition of the generated FWM products [5]. In spite of this, the simulation results agree closely with the experimental measurements, because FWM is the dominant effect. Discrepancies do not exceed $3 \mathrm{~dB}$ on the average.

For the third setup (DFRA 3), the high chromatic dispersion near the pump wavelengths produces a FWM efficiency smaller than that obtained in previous cases. In this case, figure 8 shows a better agreement between simulation results and measurements than those shown in figure 7. This is because the effects of SPM, XPM and the variations of the fiber parameters mentioned above, are not very critical in spectral regions with high chromatic dispersion.

Finally, figure 9 shows the output spectrum obtained when the DSF-LS fiber (DFRA 4) was used. Note that the output power of both pumps is higher than that measured in previous cases. The output power can be produced by the differences in attenuation, Raman gain coefficient and effective area, with respect to the other used fibers. For that reason less depletion of the pumps is produced and more pump power is available for FWM processes. As a consequence, two significant FWM products are generated from the pumps located at $1480 \mathrm{~nm}$ and 1488 $\mathrm{nm}$. We can see that the output power of these FWM products is comparable with the FWM obtained in the previous schemes in spite of the $\lambda_{Z D}$ which is located far from the pump wavelengths $\left(_{Z D}=1570 \mathrm{~nm}\right)$.

\section{FWM EFFECTS IN A GAIN-EQUALIZED DFRA}

To evaluate the effects of FWM in the spectral profile of a gain-equalized DFRA, a broadband forward DFRA is previously designed. In this case, 20 depolarized WDM channels, with an input power of $0.5 \mathrm{~mW} / \mathrm{ch}$ and placed in the range [1540-1560] $\mathrm{nm}$, are amplified along 50 $\mathrm{km}$ of TWSMF. The amplifier is designed through an optimization method based on genetic algorithms, [2] and [3], to find the wavelength and power of each pump, with the purpose of obtaining the flattest net gain in the WDM bandwidth. As a result of the design, 3 laser pumps are obtained; they are placed at $1420.28 \mathrm{~nm}, 1438.14 \mathrm{~nm}$ and $1463.52 \mathrm{~nm}$ with $160.93 \mathrm{~mW}, 92.24 \mathrm{~mW}$ and 99.61

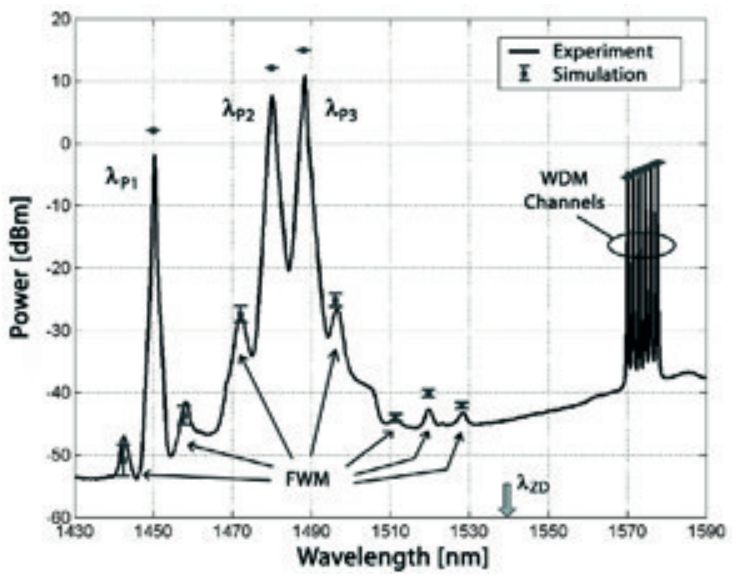

Figure 8. Simulation results vs experimental measurements. Output power spectrum of DFRA 3 setup.

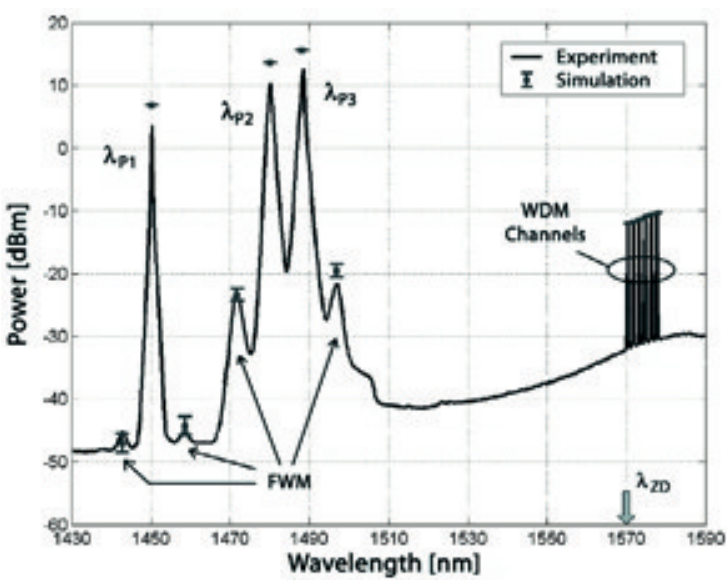

Figure 9. Simulation results vs experimental measurements. Output power spectrum of DFRA 4.

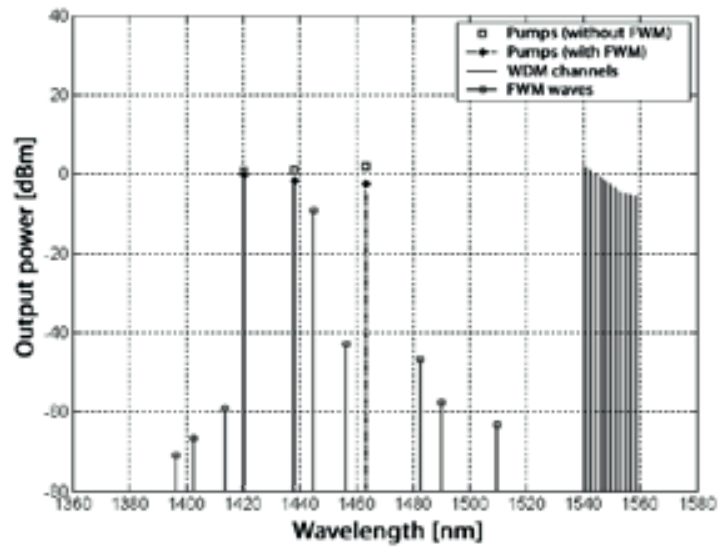

Figure 10. Output power spectrum (at $50 \mathrm{Km}$ ) of the analyzed DFRA. 
$\mathrm{mW}$ of input power, respectively. The output spectrum experiences a $0.21 \mathrm{~dB}$ ripple around $1.5 \mathrm{dBm}$ for the WDM channels.

The low chromatic dispersion of the TWSMF in the neighborhood of the pump wavelengths facilitates the pump-to-pump FWM interaction. And also because 3 pump-wavelengths are placed into the fiber several FWM products are generated. From equations (14) and (15), we can note that an interesting situation occurs when the $\lambda_{Z D}$ is located in the middle frequency of the range in which the pumps are placed, i.e., if the $\lambda_{Z D}=1441.58$ $\mathrm{nm}(208.105 \mathrm{THz})$ a non-degenerated FWM wave with unitary efficiency in $1445.03 \mathrm{~nm}$ appears (see Fig. 10). This FWM product, due to its high power and spectral position, acts as a pump for the DFRA. The new FWM products and the new SRS interactions affect the desired power equalization. In figure 11, the equalized output spectrum is compared with that obtained when FWM is included. Notice that more than $7 \mathrm{~dB}$ of ripple is obtained when FWM is undertaken. This high difference in the output power of the WDM channels produces a large penalty in the system, therefore affecting the performance of the amplifier.

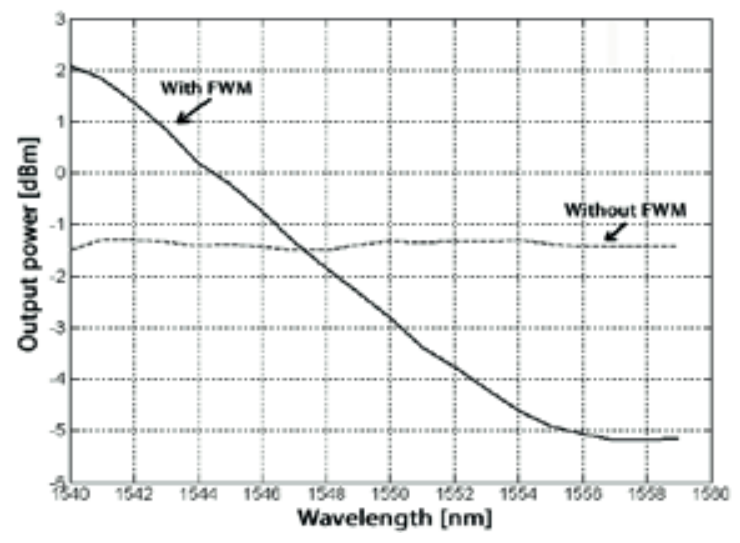

Fig. 11. Output power spectrum (at $50 \mathrm{Km}$ ) of WDM channels.

\section{CONCLUSION}

In this work a comprehensive mathematical model was proposed to describe the SRS-FWM interaction simultaneously, which can be solved by a simple numerical method. Thus, the proposed model avoids the use of iterative procedures required to obtain results when the SRS and FWM models are independently resolved. When one of the nonlinear effects (SRS or FWM) is neglected in the proposed model, the numerical results are identical to those obtained from the independent models.

On the other hand, the proposed model was validated through experimental measurements. In this way, the redistribution of the pump power along the fiber was also verified due to the highly efficient pump-to-pump FWM process (including new FWM waves, depletion and parametric gain), mainly when the dominant effects are FWM and SRS.

Then, the proposed model was used to evaluate the FWM effects in the spectral gain behavior of a DFRA. It was verified that the FWM process (including new FWM waves, depletion and parametric gain) generates new SRS interactions and a redistribution of the pump power along the fiber, causing the loss of power equalization in a gain-equalized DFRA. For that reason, the proposed model can be used to design DFRAs through some optimization method, considering the pump-to-pump FWM effects. It can be very helpful mainly when fibers with low chromatic dispersion in the pumping spectral region are used in the amplifier.

\section{ACKNOWLEDGMENT}

The authors wish to thank to $\mathrm{CPqD}$ (Campinas, Brazil), for the valuable support in the experiments. They also acknowledge the financial support for this work made by the Chilean Agency CONICYT (FONDECYT project \#1010437) and by the UTFSM project DGIP-230729.

\section{REFERENCES}

[1] M.N. Islam. "Raman Amplifiers for Telecommunications". J. Select. Topics in Quantum Electronics. Vol. 8 Nº 3, pp. 548-559. June 2002.

[2] V. Perlin and H.G. Winful. "Optimal Design of Flat-Gain Wide-Band Fiber Raman Amplifiers". J. Lightwave Technol. Vol. $20 \mathrm{~N}^{\circ}$ 2, pp. 250-254. February 2002.

[3] H. Soto, V. Pincheira and R. Olivares. "Optimal Design Based on Genetic Algorithm of Distributed Fiber Raman Amplifier Cascades". $5^{\text {th }}$ Iberoamerican Meeting on Optics and $8^{\text {th }}$ Latin American Meeting on Optics, Lasers and Their Applications. Proceedings of SPIE. Vol. 5622, pp. 364-367. SPIE. Bellingham, WA. 2004. 
[4] R.W. Freund. "Simulation of Raman amplification". Bell Laboratories Murray Hill New Jersey. 2002.

[5] G.P. Agrawal. "Nonlinear Fiber Optics". 2nd edition. Academic. San Diego, CA. 1995.

[6] J. Bouteiller, L. Leng and C. Headley. "PumpPump Four-Wave Mixing in Distributed Raman Amplified Systems". J. Lightwave Technol. Vol. 22 No 3, pp. 723-732. March 2004.

[7] F. Vanholsbeeck, S. Coen, P. Emplit, M. Haelterman and T. Sylvestre. "Coupled-mode Analysis of Stimulated Raman Scattering and Four-wave Mixing in Wavelength-Division Multiplexed Systems". Optics Communications, 250, pp. 191-201. 2005.

[8] W. Wong, C. Chen, M. Ho and H. Lee. "PhaseMatched Four-Wave Mixing Between Pumps and Signals in a Copumped Raman Amplifier". IEEE Photon. Technol. Lett. Vol. 15 N$^{\circ}$ 2, pp. 209-211. February 2003.

[9] T. Kung, C. Chang, J. Dung, and S. Chi. "Four-Wave Mixing Between Pump and Signal in a Distributed Raman Amplifier". J. Lightwave Technol. Vol. 21 $\mathrm{N}^{\mathrm{o}}$ 5, pp. 1164-1170. May. 2003.
[10] F. Di Pasquale and F. Meli. "New Raman Pump Module for Reducing Pump-Signal Four-WaveMixing Interaction in Co-Pumped Distributed Raman Amplifiers". J. Lightwave Technol. Vol. 21 No 8, pp. 1742-1748. August 2003.

[11] R.H. Stolen and J. Bjorkholm. "Parametric Amplification and Frequency Conversion in Optical Fibers". IEEE J. Quantum Electron. Vol. QE-18 No 7, pp. 1062-1072. July 1982.

[12] K. Inoue. "Polarization Effect on Four-Wave Mixing Efficiency in a Single-Mode Fiber". IEEE J. Quantum Electron. Vol. 28 No 4, pp. 883-894. April 1992.

[13] W. Wu, P. Yeh and S. Chi. "Phase Conjugation by Four-Wave Mixing in Single-Mode Fibers". IEEE Photon. Technol. Lett. Vol. 6 No 12, pp. 1448-1450. December 1994.

[14] D.L. Hart, A.F. Judy and R. Roy. "Dynamical Evolution of Multiple Four-Wave Mixing Processes in an Optical Fiber". Phys. Rev. E. Vol. $57 \mathrm{~N}^{\circ} 4$, pp. 4757-4774. April 1998. 